\title{
Classification of G-protein coupled receptors based on support vector machine with maximum relevance minimum redundancy and genetic algorithm
}

\author{
Zhanchao Li, Xuan Zhou, Zong Dai and Xiaoyong Zou*
}

\begin{abstract}
Background: Because a priori knowledge about function of G protein-coupled receptors (GPCRs) can provide useful information to pharmaceutical research, the determination of their function is a quite meaningful topic in protein science. However, with the rapid increase of GPCRs sequences entering into databanks, the gap between the number of known sequence and the number of known function is widening rapidly, and it is both time-consuming and expensive to determine their function based only on experimental techniques. Therefore, it is vitally significant to develop a computational method for quick and accurate classification of GPCRs.
\end{abstract}

Results: In this study, a novel three-layer predictor based on support vector machine (SVM) and feature selection is developed for predicting and classifying GPCRs directly from amino acid sequence data. The maximum relevance minimum redundancy (mRMR) is applied to pre-evaluate features with discriminative information while genetic algorithm (GA) is utilized to find the optimized feature subsets. SVM is used for the construction of classification models. The overall accuracy with three-layer predictor at levels of superfamily, family and subfamily are obtained by cross-validation test on two non-redundant dataset. The results are about 0.5\% to $16 \%$ higher than those of GPCR-CA and GPCRPred.

Conclusion: The results with high success rates indicate that the proposed predictor is a useful automated tool in predicting GPCRs. GPCR-SVMFS, a corresponding executable program for GPCRs prediction and classification, can be acquired freely on request from the authors.

\section{Background}

G protein-coupled receptors (GPCRs), also known as $7 \alpha$ helices transmembrane receptors due to their characteristic configuration of an anticlockwise bundle of 7 transmembrane $\alpha$ helices [1], are one of the largest superfamily of membrane proteins and play an extremely important role in transducing extracellular signals across the cell membrane via guanine-binding proteins (G-proteins) with high specificity and sensitivity [2]. GPCRs regulate many basic physicochemical processes contained in a cellular signaling network, such as smell, taste, vision, secretion, neurotransmission, metabolism, cellular

* Correspondence: ceszxy@mail.sysu.edu.cn

1 School of Chemistry and Chemical Engineering, Sun Yat-Sen University, Guangzhou 510275, PR China

Full list of author information is available at the end of the article differentiation and growth, inflammatory and immune response [3-9]. For these reasons, GPCRs have been the most important and common targets for pharmacological intervention. At present, about $30 \%$ of drugs available on the market act through GPCRs. However, detailed information about the structure and function of GPCRs are deficient for structure-based drug design, because the determination of their structure and functional using experimental approach is both time-consuming and expensive.

As membrane proteins, GPCRs are very difficult to crystallize and most of them will not dissolve in normal solvents [10]. Accordingly, the $3 \mathrm{D}$ structure of only squid rhodopsin, $\beta 1, \beta 2$ adrenergic receptor and the A2A adenosine receptor have been solved to data. In contrast, the amino acid sequences of more than 1000 GPCRs are 
known with the rapid accumulation of data of new protein sequence produced by the high-throughput sequencing technology. In view of the extremely unbalanced state, it is vitally important to develop a computational method that can fast and accurately predict the structure and function of GPCRs from sequence information.

Actually, many predictive methods have been developed, which in general, can be roughly divided into three categories. The first one is proteochemometric approach developed by Lapinsh [11]. However, the methods need structural information of organic compounds. The second one is based on similarity searches using primary database search tools (e.g. BLAST, FASTA) and such database searches coupled with searches of pattern databases (PRINTS) [12]. However, they do not seem to be sufficiently successful for comprehensive functional identification of GPCRs, since GPCRs make up a highly divergent family, and even when they are grouped according to similarity of function, their sequences share strikingly little homology or similarity to each other [13]. The third one is based on statistical and machine learning method, including support vector machines (SVM) [8,14-17], hidden Markov models (HMMs) $[1,3,6,18]$, covariant discriminant (CD) $[7,11,19,20]$, nearest neighbor (NN) $[2,21]$ and other techniques [13,22-24].

Among them, SVM that is based on statistical learning theory has been extensively used to solve various biological problems, such as protein secondary structure $[25,26]$, subcellular localization $[27,28]$, membrane protein types [29], due to its attractive features including scalability, absence of local minima and ability to condense information contained in the training set. In SVM, an initial step to transform protein sequence into a fixed length feature vector is essential because SVM can not be directly applied to amino acid sequences with different length. Two commonly used feature vectors to predict GPCRs functional classes are amino acid composition (AAC) and dipeptide composition (DipC) $[2,7,10,16,19,20,22]$, where every protein is represented by 20 or 400 discrete numbers. Obviously, if one uses AAC or DipC to represent a protein, many important information associated with the sequence order will be lost. To take into account the information, the so-called pseudo amino acid composition (PseAA) was proposed [30] and has been widely used to GPCRs and other attributes of protein studies [10,3136]. However, the existing methods were established only based on a single feature-set. And, few works tried to research the relationship between features and the functional classes of protein [37-39], or to find the informative features which contribute most to discriminate functional types. Karchin et al [8] also indicated that the performance of SVM could be further improved by using feature vector that posses the most discriminative information. Therefore, feature selection should be used for accurate SVM classification.

Feature selection, also known as variable selection or attribute selection, is the technique commonly used in machine learning and has played an important role in bioinformatics studies. It can be employed along with classifier construction to avoid over-fitting, to generate more reliable classifier and to provide more insight into the underlying causal relationships [40]. The technique has been greatly applied to the field of microarray and mass spectra (MS) analysis [41-50], which has a great challenge for computational techniques due to their high dimensionality. However, there is still few works utilizing feature selection in GPCRs prediction to obtain the most informative features or to improve the prediction accuracy.

So, a new predictor combining feature selection and support vector machine is proposed for the identification and classification of GPCRs at the three levels of superfamily, family and subfamily. In every level, minimum redundancy maximum relevance (mRMR) [51] is utilized to pre-evaluate features with discriminative information. After that, to further improve the prediction accuracy and to obtain the most important features, genetic algorithms (GA) [52] is applied to feature selection. Finally, three models based on SVM are constructed and used to identify whether a query protein is GPCR and which family or subfamily the protein belongs to. The prediction quality evaluated on a non-redundant dataset by the jackknife cross-validation test exhibited significant improvement compared with published results.

\section{Methods}

Dataset

As is well-known, sequence similarity in dataset has an important effect on the prediction accuracy, i.e. accuracy will be overestimated when using high similarity protein sequence. Thus, in order to disinterestedly test current method and facilitate to compare with other existing approaches, the dataset constructed by Xiao [10] is used as the working dataset. The similarity in the dataset is less than $40 \%$. The dataset contains 730 protein sequences that can be classified into two parts: 365 non-GPCRs and 365 GPCRs. The 365 GPCRs can be divided into 6 families: 232 rhodopsin-like, 44 metabotropic glutamate/ pheromone, 39 secretin-like, 23 fungal pheromone, 17 frizzled/smoothened and 10 cAMP receptor. For rhodopsin-like of GPCRs, we further partitioned into 15 subfamilies based on GPCRDB (release 10.0) [53], including 46 amine, 72 peptide, 2 hormone, 17 rhodopsin, 19 olfactory, 7 prostanoid, 13 nucleotide, 2 cannabinoid, 1 plate- 
let activating factor, 2 gonadotropin-releasing hormone, 3 thyrotropin-releasing hormone \& secretagogue, 2 melatonin, 9 viral, 4 lysosphingolipid, 2 leukotriene B4 receptor and 31 orphan. Those subfamilies, which the number of proteins is lower than 10, are combined into a class, because they contain too few sequences to have any statistical significance. So, 6 classes (46 amine, 72 peptide, 17 rhodopsin, 19 olfactory, 13 nucleotide and 34 other) are obtained at subfamily level.

\section{Protein represent}

In order to fully characterize protein primary structure, 10 feature vectors are employed to represent the protein sample, including AAC, DipC, normalized Moreau-Broto autocorrelation (NMBAuto), Moran autocorrelation (MAuto), Geary autocorrelation (GAuto), composition (C), transition (T), distribution (D) [54], composition and distribution of hydrophobicity pattern (CHP, DHP). Here 8 and 7 amino acid properties extracted from AAIndex database [55] are selected to compute autocorrelation, C, $\mathrm{T}$ and $\mathrm{D}$ features, respectively. The properties and definitions of amino acids attributed to each group are shown in Additional file 1 and 2.

According to the theory of Lim [56], 6 kinds of hydrophobicity patterns include: $(i, i+2),(i, i+2, i+4),(i, i+3),(i$, $i+1, i+4),(i, i+3, i+4)$ and $(i, i+5)$. The patterns $(i, i+2)$ and $(i, i+2, i+4)$ often appear in the $\beta$-sheets while the patterns $(i, i+3),(i, i+1, i+4)$ and $(i, i+3, i+4)$ occur more often in $\alpha$-helices. The pattern $(i, i+5)$ is an extension of the concept of the "helical wheel" or amphipathic $\alpha$-helix [57]. Seven kinds of amino acids, including Cys (C), Phe $(\mathrm{F})$, Ile (I), Leu (L), Met (M), Val (V) and Trp (W), may occur in the 6 patterns based on the observed of Rose et al [58]. Because transmembrane regions of membrane protein are usually composed of $\beta$-sheet and $\alpha$-helix, CHP and DHP are used to represent protein sequence.

For the pattern $(i, i+2)$, the CHP is computed by Eq. (1):

$$
C H P(i, i+2)=\frac{N(i, i+2)}{L-2} i=1,2, \ldots \ldots, L-2
$$

Where, $N_{(i, i+2)}$ is the number of pattern in position $i$ and $i+2$ that simultaneously belong to any of 7 kinds of amino acids, and $L$ is the sequence length. Other CHP are calculated by using the rule mentioned above. The DHP of pattern $(i, i+2)$, which describes the distribution of the pattern in protein sequence, can be calculated according to Eq. (2):

$$
\operatorname{DHP}(i, i+2)=\frac{S(i, i+2)}{L-2} i=1,2, \ldots \ldots, L-2
$$

Where, $S_{(i, i+2)}$ is a feature vector composing of 5 values that are the position in the whole sequence for the first pattern $(i, i+2), 25 \%$ patterns $(i, i+2), 50 \%$ patterns $(i, i+2)$, $75 \%$ patterns $(i, i+2)$ and $100 \%$ patterns $(i, i+2)$, respectively. How to calculate these values is explained below by using a random sequence with 40 amino acids, as shown in Figure 1, which consists of 10 patterns $(i, i+2)$. The 10 patterns $(i, i+2)$ included, CAL (1), IQF (2), FKM (3), MDV (4), CTF (5), FYL (6), CFM (7), FMI (8), IRI (9), CAW (10). The first pattern $(i, i+2)$ is in the pattern position of $1(\mathrm{CAL})$. The pattern $(i, i+2)$ of $(10 \times 25 \%=2.5 \approx 3)$ is in the pattern position of 3 (FKM). The pattern $(i, i+2)$ of $(10 \times 50 \%=5)$ is in the pattern position of 5 (CTF). The pattern $(i, i+2)$ of $(10 \times 75 \%=7.5 \approx 8)$ is in the pattern position of 8 (FMI). The pattern $(i, i+2)$ of $(10 \times 100 \%=$ $10)$ is in the pattern position of $10(\mathrm{CAW})$. The first letter of the 5 patterns $(i, i+2)$ are $\mathrm{C}, \mathrm{F}, \mathrm{C}, \mathrm{F}, \mathrm{C}$, which is corresponding to the residue position of $1,10,17,28$, and 36 in the sequence, respectively. Thus, $S_{(i, i+2)}=\left[\begin{array}{lllll}1 & 10 & 17 & 28 & 36\end{array}\right]$.
Sequence

Sequence index

1

10

20

30

Hydrophobic pattern

$(\mathrm{i}, \mathrm{i}+2)$ index

1

$\begin{array}{lll}2 & 3 & 4\end{array}$

56

789

10

Figure 1 Random sequence consisting of 40 residues as an example to illustrate derivation of feature vector 
Similarly, the DHP for pattern other than $(i, i+2)$ is also calculated by using the rule.

\section{The optimized feature subset selection}

SVM is one of the most powerful machine learning methods, but it cannot perform automatic feature selection. To overcome this limitation, various feature selection methods were introduced $[59,60]$. Feature selection methods typically were divided into two categories: filter and wrapper methods. Although filter methods are computationally simple and easily scale to high-dimensional dataset, they ignore the interaction between selected feature and classifier. In contrast, wrapper approaches include the interaction and can also take into account the correlation between features, but they have a higher risk of overfitting than filter techniques and are very computationally intensive, especially if building the classifier has a high computational cost [61]. Considering the characteristics of the two methods, the mRMR belonging to filter methods is used to preselect a feature subset, and then GA belonging to wrapper methods is utilized to obtain the optimized feature subset.

\section{Minimum redundancy maximum relevance (mRMR)}

The mRMR method tries to select a feature subset, each of which has the maximal relevance with target class and the minimal redundancy with other features. The feature subset can be obtained by calculating the mutual information between the features themselves and between the features and the class variables. In the current study, feature is a vector contains 10 type descriptors values of proteins (AAC, DipC, NMBAuto, MAuto, GAuto, C, T, D, $\mathrm{CHP}$ and DHP). For binary classification problem, classification variable $l_{k} 1$ or 2 . The mutual information $M I(x, y)$ of between two features $x$ and $y$ is computed by Eq.(3):

$$
M I(x, y)=\sum_{i, j \in N} p\left(x_{i}, y_{j}\right) \log \frac{p(x i, y j)}{p(x i) p(y j)}
$$

Where, $p\left(x_{i} y_{j}\right)$ is joint probabilistic density, $p\left(x_{i}\right)$ and $p\left(y_{j}\right)$ is marginal probabilistic density.

Similarly, the mutual information $M I(x, l)$ of between classification variable $l$ and feature $x$ is also calculated by Eq.(4):

$$
M I(x, l)=\sum_{i, k \in N} p\left(x_{i}, l_{k}\right) \log \frac{p(x i, l k)}{p(x i) p(l k)}
$$

The minimum redundancy condition is to minimize the total redundancy of all features selected by Eq.(5):

$$
\min (m R), m R=\frac{1}{|S|^{2}} \sum_{x, y \in S} M I(x, y)
$$

Where, $S$ denoted that the feature subset, and $|S|$ is the number of feature in $S$.

The maximum relevance condition is to maximize the total relevance between all features in $S$ and classification variable. The condition can be obtained by Eq. (6):

$$
\max (M R), M R=\frac{1}{|S|} \sum_{x \in S} M I(x, l)
$$

To achieve feature subset, the two conditions should be optimized simultaneously according to Eq. (7):

$$
\max \left(\nabla_{M I}\right), \nabla_{M I}=M R-m R
$$

If continuous features exist in feature set, the feature must be discretized by using "mean \pm standard deviation/ 2 " as boundary of the 3 states. The value of feature larger than "mean + standard deviation/2" is transformed to state 1 ; The value of feature between "mean - standard deviation/2" and "mean + standard deviation/2" is transformed to state 2; The value of feature smaller than "mean - standard deviation/2" is transformed to state 3 . In this case, computing mutual information is straightforward, because both joint and marginal probability tables can be estimated by tallying the samples of categorical variables in the data [51]. More explanation about the calculation of probability can be seen from Additional file 3 . Detailed depiction of the mRMR method can be found in reference [51], and mRMR program can be obtained from http://penglab.janelia.org/proj/mRMR/index.htm

\section{Genetic algorithms (GA)}

GA can effectively search the interesting space and easily solve complex problems without requiring a prior knowledge about the space and the problem. These advantages of GA make it possible to simultaneously optimize the feature subset and the SVM parameters. The chromosome representations, fitness function, selection, crossover and mutation operator in GA are described in the following sections.

\section{Chromosome representation}

The chromosome is composed of decimal and binary coding systems, where binary genes are applied to the selection of features and decimal genes are utilized to the optimization of SVM parameters.

\section{Fitness function}

In this study, two objectives must be simultaneously considered when designing the fitness function. One is to 
maximize the classification accuracy, and the other is to minimize the number of selected features. The performances of these two objectives can be evaluated by Eq. (8):

$$
\text { fitness=SVM_accuracy+(1-n/N) }
$$

Where, $S V M$ _ accuracy is SVM classification accuracy, $n$ is the number of selected features, $N$ is the number of overall features.

\section{Selection, crossover and mutation operator}

Elitist strategy that guarantees chromosome with the highest fitness value is always replicated into the next generation is used to select operation. Once a pair of chromosome is selected for crossover, five random selected positions are assigned to the crossover operator of the binary coding part. The crossover operator was determined according to Eq. (9) and (10) for the decimal coding part, where $p$ is the random number of $(0,1)$.

$$
\begin{aligned}
& \text { child } 1=p^{*} \text { parent } 1+(1-p)^{*} \text { parent } 2 \\
& \text { child } 2=p^{*} \text { parent } 2+(1-p)^{*} \text { parent } 1
\end{aligned}
$$

The method based on chaos [62] is applied to the mutation operator of decimal coding. Mutation to the part of binary coding is the same as traditional GA.

The population size of GA is 30 , and the termination condition is that the generation numbers reach 10000. A detailed depiction of the GA can be reference to our previous works [63].

\section{Model construction and assessment of performance}

For the present SVM, the publicly available LIBSVM software [64] is used to construct the classifier with the radial basis function as the kernel. Ten-fold cross-validation test is used to examine a predictor for its effectiveness. In the 10 -fold cross-validation, the dataset is divided randomly into 10 equally sized subsets. The training and testing are carried out 10 times, each time using one distinct subset for testing and the remaining 9 subsets for training.

Classifying GPCRs in superfamily level can be formulated as a binary classification problem, namely each protein can be classified as either GPCRs or non-GPCRs. So, the performance of classifier are measured in terms of sensitivity (Sen), specificity (Spe), accuracy (Acc) and Matthew's correlation coefficient (MCC) [65], and are given by Eqs. (11)-(14).

$$
\operatorname{Sen}=\frac{T P}{T P+F N}
$$

$$
\begin{aligned}
& S p e=\frac{T N}{T N+F P} \\
& A c c=\frac{T P+T N}{T P+T N+F P+F N} \\
& M C C= \\
& \frac{T P \cdot T N-F P \cdot F N}{\sqrt{(T P+F N) \cdot(T P+F P) \cdot(T N+F N) \cdot(T N+F P)}}
\end{aligned}
$$

Here, TP, TN, FP and $F N$ are the numbers of true positives, true negatives, false positives and false negatives, respectively.

The classification of GPCRs into its families and subfamilies is a multi-class classification problem, namely a given protein can be classified into specific family or subfamily. The simple solution is to reduce the multi-classification to a series of binary classifications. We adopted the one-versus-one strategy to transfer it into a series of twoclass problems. The overall accuracy $(Q)$ and accuracy $\left(Q_{i}\right)$ for each family or subfamily calculated for assessment of the prediction system are given by Eqs. (15)-(16).

$$
\begin{aligned}
& Q=\frac{\sum_{i=1}^{k} p(i)}{N} \\
& Q_{i}=\frac{p(i)}{o b s(i)}
\end{aligned}
$$

Where, $N$ is the total number of sequences, $o b s(i)$ is the number of sequences observed in class $i, p(i)$ is the number of correctly predicted sequences of class.

The whole procedure for recognizing GPCRs form protein sequences and further classifying GPCRs to family and subfamily is illustrated in Figure 2, and the steps are as follows:

Step 1. Produce various feature vectors that represent a query protein sequence.

Step 2. Preselect a feature subset by running mRMR. Select an optimized feature subset from the preselect subset by GA and SVM. Predict whether the query protein belong to the GPCRs or not. If the protein is classified into non-GPCRs, stop the process and output results, otherwise, go to the next step.

Step 3. Preselect again a feature subset and further select an optimized feature subset. Predict which family the protein belongs. If the protein is divided into nonRhodopsin like, stop the process with the output of results, otherwise, go to the next step. 


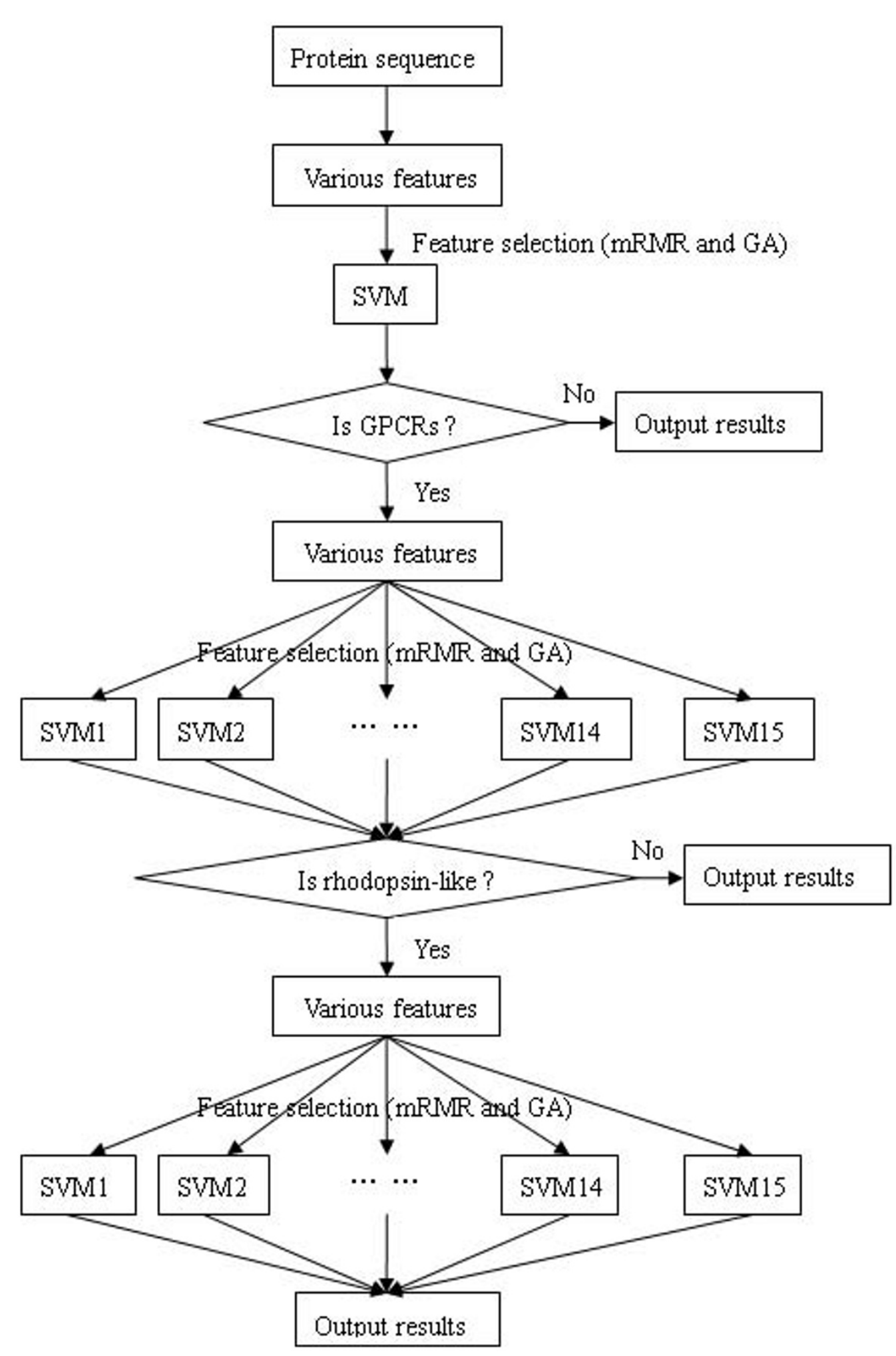


Step 4. Preselect a feature subset again and select an optimized feature subset. Predict which subfamily the protein belongs to.

\section{Results and discussion Identification a GPCR from non-GPCR}

At the first step of feature selection, only 600 different feature subsets are selected based on mRMR due to our limited computational power, and the feature subsets contains $1,2,3, \ldots . ., 598,599$ and 600 features respectively. The performance of various feature subsets for discriminating between GPCRs and other protein is investigated based on grid search for maximal 10-fold cross-validation tested accuracy with $\gamma$ ranging among $2^{-}$ $5,2^{-4}, \ldots, 2^{15}$, and $C$ ranging among $2^{-15}, 2^{-14}, \ldots, 2^{5}(\gamma$ and $C$ are needed to optimize parameters of SVM), and the results are shown in Figure 3. The accuracy for a single feature is $85.89 \%$. And the accuracy dramatically increased when the number of features increased from 2 to 150 , and achieved the highest values $(98.22 \%)$ while the feature subset consists of 543 features. However, the accuracy did not change dramatically when the number of features increased to 600 .

Although the highest accuracy can be obtained by using the feature subset with 543 features, many features impede the discovery of physicochemical properties that affect the prediction of GPCRs. So, we perform further GA for the preselecting feature subset that consists of 600 features. Figure 4 and Figure 5 illustrate the convergence processes for GA to select feature subset. Initially, approximate 275 features are selected by GA and a predictive accuracy about $94.93 \%$ is achieved based on 10 fold cross-validation tested. Along with the implementation of GA, the number of selected features gradually decreased while fitness is improved. Finally, the good fitness, high classification accuracy $(98.77 \%$ based on 10 fold cross-validation test) and optimized feature subset (only contains 38 features) can be obtained from about 6600 generations. Consequently, the optimal classifier at superfamily level is constructed with the optimal feature subset.

The results of the optimized features subset are shown in Figure 6. The optimized features subset contains 38 features, including 1 feature of cysteine composition; 7 features of DipC based on Phe-Phe, Gly-Glu acid, HisAsp, Ile-Glu, Asn-Ala, Asn-Met and Ser-Glu; 1 feature of $\mathrm{C}$ based on polarity grouping; 2 features of $\mathrm{T}$ based on hydrophobicity and buried volume grouping; 7 features of D based on charge, hydrophobicity, Van der Waals volume, polarizability and solvent accessibility grouping; 5 features of NMBAuto based on hydrophbicity, flexibility, residue accessible surface area and relative mutability; 11 features of MAuto based on hydrophobicity, flexibility, residue volume, steric parameter and relative mutability; 2 features of GAuto based on hydrophobicity and free energy; 2 features of DHP based on pattern $(i, i+3, i+4)$. The results suggest that the order of these feature groups that contributed to the discrimination GPCRs from nonGPCRs is: MAuto > Dipc and D > NMBAuto > T, GAuto and DHP > AAC and C.

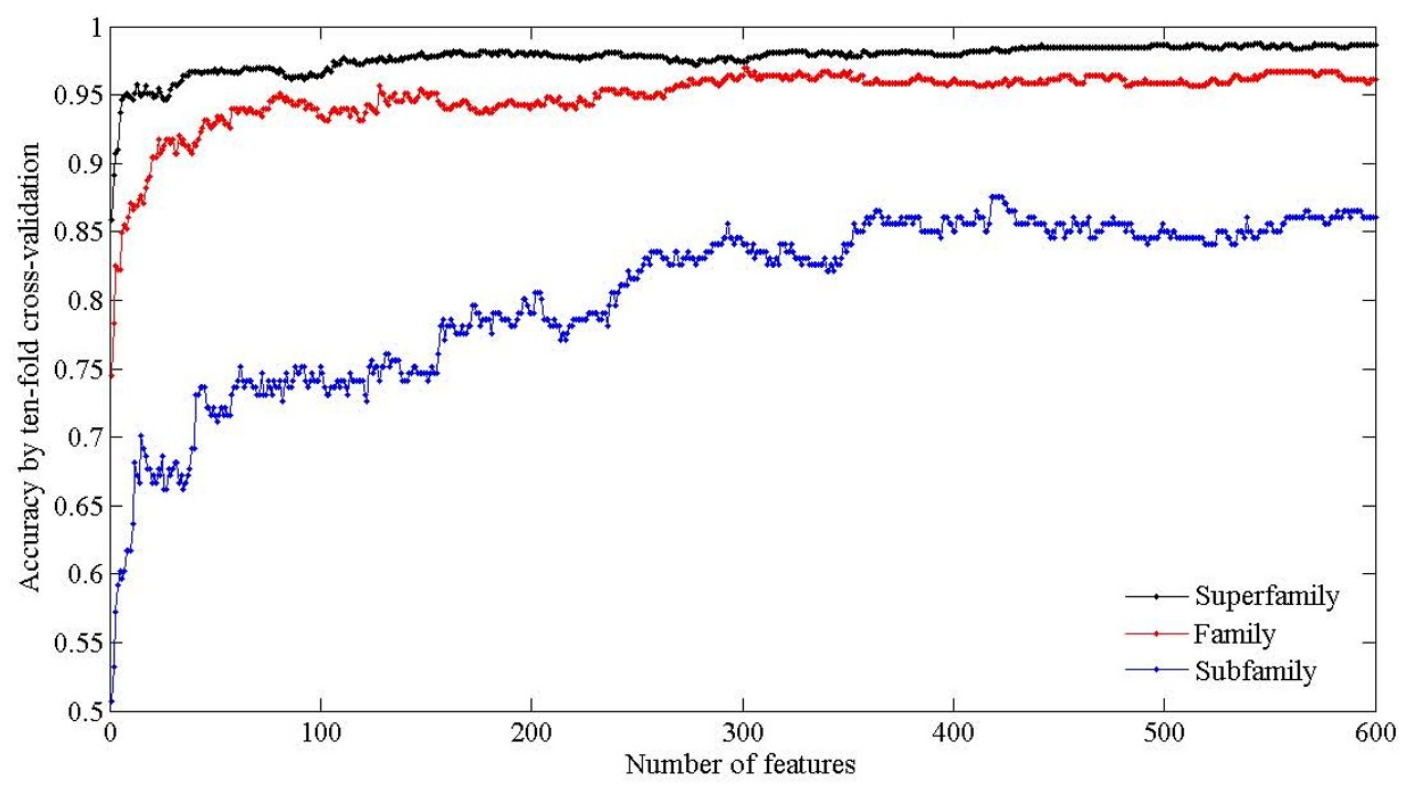

Figure 3 The relationship between the accuracy and the number of features 


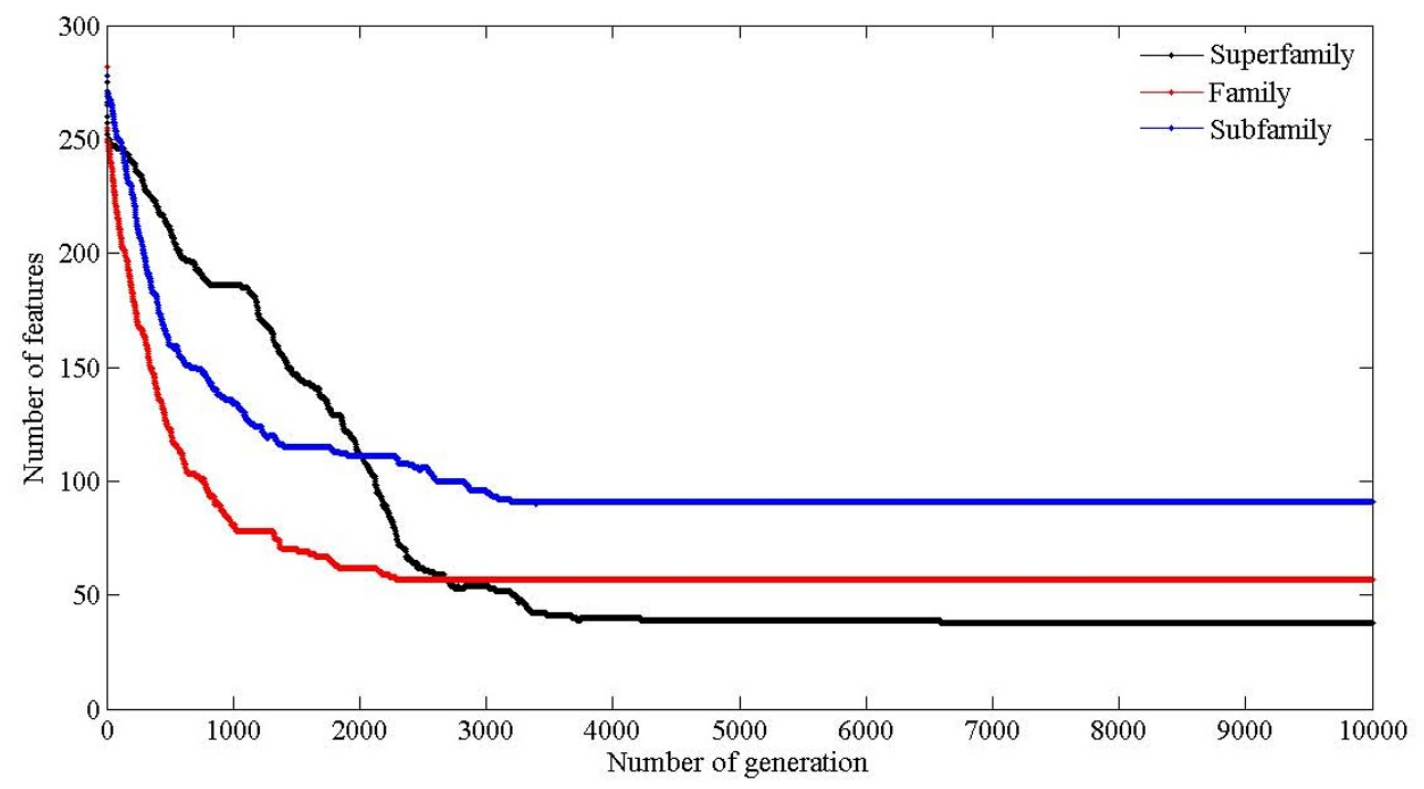

Figure 4 The relationship between the number of features and the number of generations.

\section{Recognition of GPCR family}

Following the same steps described above, the quality of various feature subsets are investigated at family level based on grid search and 10-fold cross-validation tested. The relationship between number of feature and overall accuracy is shown in Figure 3. A significant increase in overall accuracy can be observed when the number of feature increased from 1 to 301, and the highest overall accuracy of $96.99 \%$ can be achieved.

We also further perform GA for preselecting feature subset with 600 features to acquire an optimized feature subset. The processes of optimization are displayed in Figure 4 and Figure 5. It can be observed that the number of features dramatically decreased from 250 to 57 when

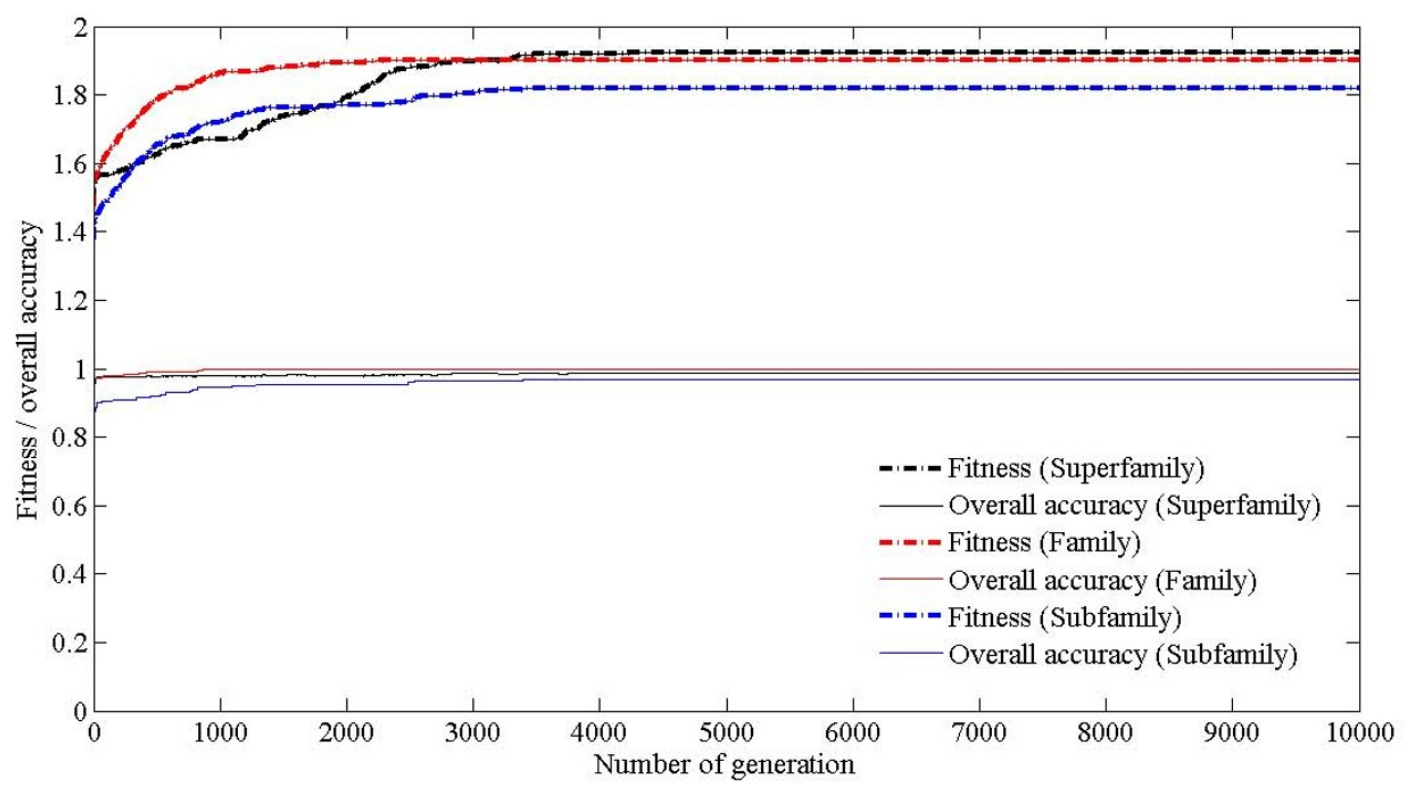

Figure 5 Fitness values and overall accuracy based on the most fitted member of each generation. 


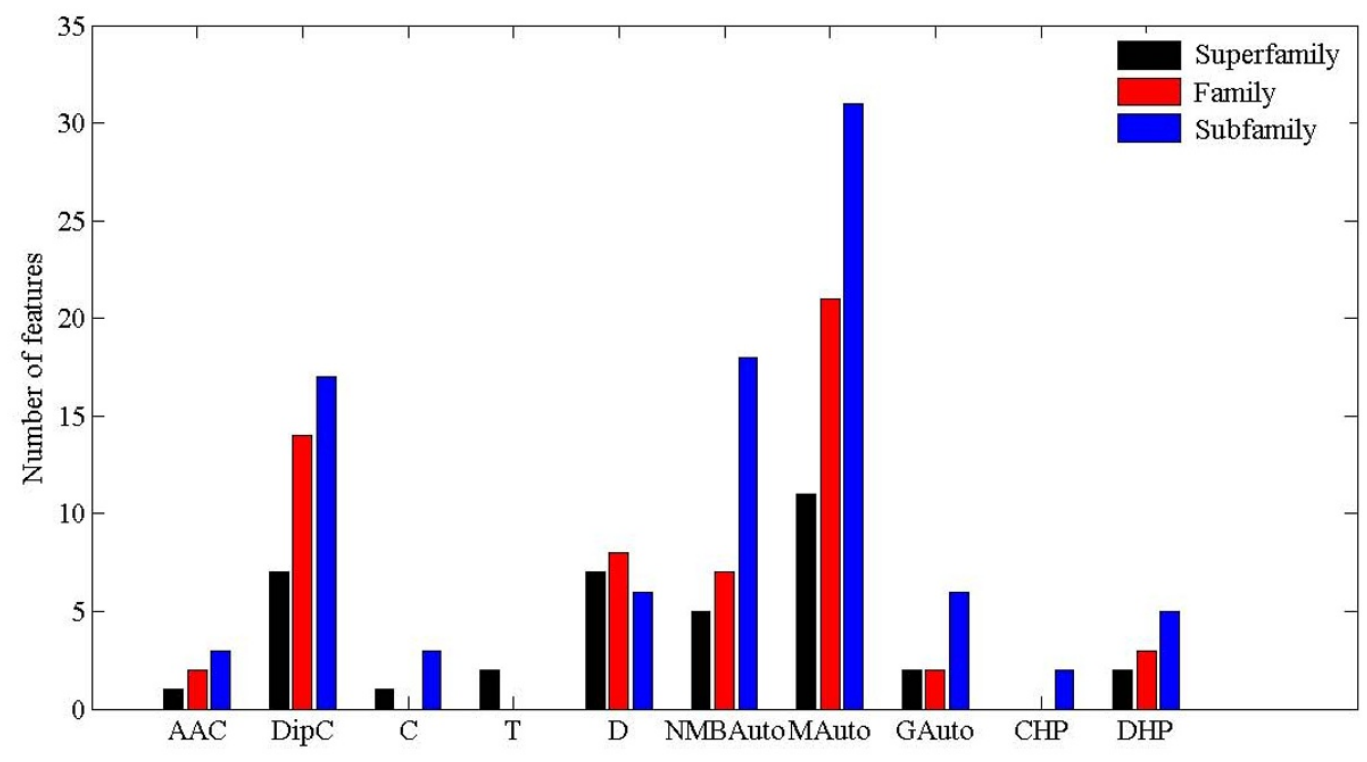

Figure 6 Composition of the optimized features subset.

the number of generation increased from 1 to 2300, and the best fitness and highest overall accuracy of $99.73 \%$ can be achieved. So, the optimal classifier with 57 features is used to construct classifier at family level.

The results of the optimized feature subset are also shown in Figure 6. The optimized features subset contains 2 AAC, 14 DipC, 8 D, 7 NMBAuto, 21 MAuto, 2 GAuto and 3 DHP features. The results reveal that the order of these feature groups that contributed to the classification GPCRs into 6 families is: MAuto $>\mathrm{DipC}>\mathrm{D}>$ NMBAuto > DHP > AAC and GAuto.

\section{Classification of GPCR subfamily}

Because knowledge of GPCRs subfamilies can provide useful information to pharmaceutical companies and biologists, the identification of subfamilies is a quite meaningful topic in assigning a function to GPCRs. Therefore, we constructed a classifier at subfamily level to predict the subfamily belonging to the rhodopsin-like family. Rhodopsin-like family is considered because it covers more than $80 \%$ of sequences in the GPCRDB database [53], and the number of other family in current dataset is too few to have any statistical significance. Similarly, we also study the quality of various feature subsets from mRMR based on grid search and 10-fold cross-validation tested. The correlation between number of features and overall accuracy is also illustrated in Figure 3. Overall accuracy enhanced when the number of features increased from 1 to 300, and the highest overall accuracy of $87.56 \%$ can be obtained by using the feature subset with 418 features.
In order to get an optimized feature subset, GA is further applied to further feature selection from a preselected feature subset with 600 features. The processes of convergence are shown in Figure 4 and Figure 5 . The number of features in optimized feature subset significantly decreased from 278 to 115 when the number of generation increased from 1 to 1400, and corresponding fitness value is significantly increased. Subsequently, the number of features and fitness value maintained invariable. It clearly shows a premature convergence. However, the number of features decreased from 113 to 92 when the number of generation increased from 1800 to 3100 , indicating GA has ability to escape from local optima. The finally optimized feature subset with 91 features can be obtained within 3200 generations. Therefore, we developed a classifier by the features from the optimized feature subset for classifying the subfamilies of the rhodopsin-like family.

The composition of optimized feature subset is shown in Figure 6. The optimized feature subset contains 3 AAC, 17 DipC, 3 C, 6 D, 18 NMBAuto, 31 MAuto, 6 GAuto, $2 \mathrm{CHP}$ and $5 \mathrm{DHP}$ features. The results suggest that the order of these feature groups that contributed to the prediction subfamily belonging to the rhodopsin-like family is: MAuto > NMBAuto $>$ DipC $>$ D and GAuto > DHP > AAC and C > CHP.

\section{Comparison with GPCR-CA}

To facilitate a comparison with GPCR-CA method developed by Xiao [10], we perform jackknife cross-validation test based on the current predictor. GPCR-CA is a two- 
layer classifier that is used to classify at the levels of superfamily and family, respectively, and each protein is characterized by PseAA, which is based on "cellular automation" and gray-level co-occurrence matrix factors. In the jackknife test, each protein in the dataset is in turn singled out as an independent test sample and all the rule-parameters are calculated without using this protein. The results of jackknife test obtained with proposed method in comparison with GPCR-CA are listed in Table 1 and Figure 7. The performances of the proposed predictor (GPCR-SVMFS) in predicting the subfamilies are summarized in Table 2.

It can be seen from Table 1 that the accuracy, sensitivity, specificity and MCC by GPCR-SVMFS are $97.81 \%$, $97.04 \%, 98.61 \%$ and 0.9563 , respectively, which are $4.7 \%$ to $7.6 \%$ improvement over GPCR-CA method [10]. The results indicated that the GPCR-SVMFS can identify GPCRs from non-GPCRs with high accuracy using optimized feature subset as the sequence feature.

As can be seen from Figure 7, the overall accuracy of GPCR-SVMFS is $99.18 \%$, which is almost $15 \%$ higher than that of GPCR-CA. Furthermore, the accuracies of fungal pheromone, cAMP and frizzled/smoothened family are dramatically improved. The accuracy by GPCRSVMFS for fungal pheromone family is $100 \%$, approximately $93 \%$ higher than the accuracy by the GPCR-CA. The accuracies of cAMP and frizzled/smoothened are $100 \%$ and $94.12 \%$ based on GPCR-SVMFS, approximately $40 \%$ and $47 \%$ higher than the accuracy by the GPCR-CA, respectively. In additional, as for secretin and metabotropic glutamate/pheromone family, the predictive accuracies are $97.44 \%$ and $97.73 \%$ by GPCR-SVMFS, approximately $23 \%$ and $16 \%$ higher than those of GPCRCA, indicating GPCR-SVMFS is effective and helpful for the prediction of GPCRs at family level.

As shown in Table 2, the accuracies of amine, peptide, rhodopsin, olfactory and other are $93.48 \%, 98.61 \%$, $88.24 \%$ and $94.12 \%$, respectively. Meanwhile, we also have notice that the accuracy of nucleotide is lower than that of amine, peptide, rhodopsin, olfactory, which may be caused by the less protein samples contained in nucleotide class. Although the accuracy for nucleotide is only $76.92 \%$, the overall accuracy is $94.53 \%$ for identifying subfamiliy, indicating the current method can yield quite reliable results at subfamily level.

\section{Comparison with GPCRPred}

Furthermore, in order to roundly evaluate our method we also performed it on another dataset used in GPCRPred [14], which is a three-layer classifier based on SVM. In the classifier, DipC is used for characterizing GPCRs at the levels of superfamily, family and subfamily. The dataset obtained from GPCRPred contains 778 GPCRs and 99 non-GPCRs. The 778 GPCRs can be divided into 5 fami- lies: 692 class A-rhodopsin and andrenergic, 56 class Bcalcitonin and parathyroid hormone, 16 class Cmetabotropic, 11 class D-pheromone and 3 class EcAMP. The class A at subfamily level is composed of 14 major classes and sequences are from the work of Karchin [8].

The success rates are listed in Tables 3, 4 and 5. And the results of GPCR-SVMFS are compared with those of GPCRPred for the same dataset. From Table 3 we can see that the accuracy of GPCR-SVMFS is $0.5 \%$ higher than that of GPCRPred based on DipC at superfamily level. As can be seen from Table 4, the accuracies for class A, class B and class C are $100 \%$, which is almost $2 \%, 15 \%$ and $19 \%$ higher than that of GPCRPred, respectively. Especially for the class $\mathrm{D}$, the predictive accuracy is improved to $81.82 \%$ by GPCR-SVMFS, which is almost $45 \%$ higher than that of GPCRPred. As can be seen in Table 5, the accuracies of the nucleotide, viral and lysospingolipids are improved to $93.75 \%, 76.47 \%, 100.0 \%$, about $8 \%, 43 \%$ and $42 \%$ higher than GPCRPred. Although the accuracy of cannabis is decreased from $100 \%$ to $90.91 \%$, the overall accuracy is improved from $97.30 \%$ to $98.77 \%$. All the results show that GPCR-SVMFS is superior to GPCRPred, which may be caused by the fact that optimized feature subset contains more information than single DipC, and therefore can enhance predictive performance significantly.

\section{Predictive power of GPCR-SVMFS}

In order to test the performance of GPCR-SVMFS to identify orphan GPCRs, a dataset (we called it as "deorphan") containing 274 orphan proteins are collected from the GPCRDB database (released on 2006). We further verify the 274 orphan proteins by searching accession number in the latest version of GPCRDB (released on 2009). The results indicated that 8 proteins, 19 proteins and 2 proteins belong to amine, peptide and nucleotide respectively. Finally, the dataset of 29 proteins is constructed (The dataset can be obtained from Additional file 4.

The GPCR-SVMFS is able to accurately identify 13 peptides from 19 proteins, and 2 nucleotides are completed recognized. However, none of the 8 amines is correctly identified. So, overall success rate is $19 / 29=51.72 \%$. The result is higher than that of completely randomized prediction, because the rate of correct identification by randomly assignment is $1 / 6=16.67 \%$ if the protein samples are completely randomly distributed among the 6 possible subfamilies (i.e. amine, peptide, rhodopsin, olfactory, nucleotide and other). The results imply that GPCRSVMFS is indeed powerful to identify orphan GPCRs.

In addition, the prediction power of GPCR-SVMFS is also evaluated at family level and subfamily level by using 8 independent dataset, which are collected based on the GPCRDB (released on 2009). Three of the 8 dataset at 
Table 1: Comparison of different method by the jackknife at superfamily level

\begin{tabular}{ccccc}
\hline Method & Acc(\%) & Sen(\%) & Spe(\%) & MCC \\
\hline GPCR-CA [10] & 91.46 & 92.33 & 90.96 & N/A \\
GPCR-SVMFS & 97.81 & 97.04 & 98.61 & 0.9563 \\
\hline
\end{tabular}

family level are rhodopsin-like, metabotropic and secretin-like, which contains 20290, 1194 and 1484 proteins, respectively. Other 5 dataset at subfamily level are amine, peptide, rhodopsin, olfactory and nucleotide. The 5 dataset is composed of 1840, 4169, 1376, 9977 and 576 proteins, respectively ( 8 dataset are given in Additional file 5, $6,7,8,9,10,11,12$ ).

The results at family level are shown in Table 6 . The proposed method achieves accuracy of $96.16 \%$ for rhodopsin-like, $85.76 \%$ for metabotropic and $68.53 \%$ for secretin-like, and an overall accuracy of $93.81 \%$ can also be obtained. The results indicate that the performance of GPCR-SVMFS is good enough at family level.

The results for 5 subfamilies are listed in Table 7. The prediction accuracies for the rhodopsin, amine and peptide reach $87.79 \%, 80.22 \%$ and $74.12 \%$, respectively. For the largest subfamily (olfactory) that contains 9977 proteins, the accuracy achieves the highest values of $90.96 \%$. Although the accuracy for nucleotide is only $54.69 \%$, the overall prediction accuracy achieves $84.54 \%$ for classifying subfamily, indicating the GPCR-SVMFS method can yield good results at subfamily level.
Table 2: Success rates obtained with the GPCR-SVMFS predictor by jackknife test at subfamily level

\begin{tabular}{cccc}
\hline $\begin{array}{c}\text { GPCR } \\
\text { subfamily }\end{array}$ & $\begin{array}{c}\text { Number } \\
\text { of proteins }\end{array}$ & $\begin{array}{c}\text { Number of } \\
\text { correct } \\
\text { prediction }\end{array}$ & $\mathbf{Q}_{i} / \mathbf{Q}(\%)$ \\
\hline Amine & 46 & 43 & 93.48 \\
Peptide & 72 & 71 & 98.61 \\
Rhodopsin & 17 & 15 & 88.24 \\
Olfactory & 19 & 19 & 100.0 \\
Nucleotide & 13 & 10 & 76.92 \\
Other & 34 & 32 & 94.12 \\
\hline Overall & 201 & 190 & 94.53 \\
\hline
\end{tabular}

\section{Conclusion}

With the rapid increment of protein sequence data, it is indispensable to develop an automated and reliable method for classification of GPCRs. In this paper, a threelayer classifier is proposed for GPCRs by coupling SVM with feature selection method. Compared with existing methods, the proposed method provides better predictive performance, and high accuracies for superfamily, family and subfamily of GPCRs in jackknife cross-validation test, indicating the investigation of optimized features subset are quite promising, and might also hold a potential as a useful technique for the prediction of other attributes of protein.

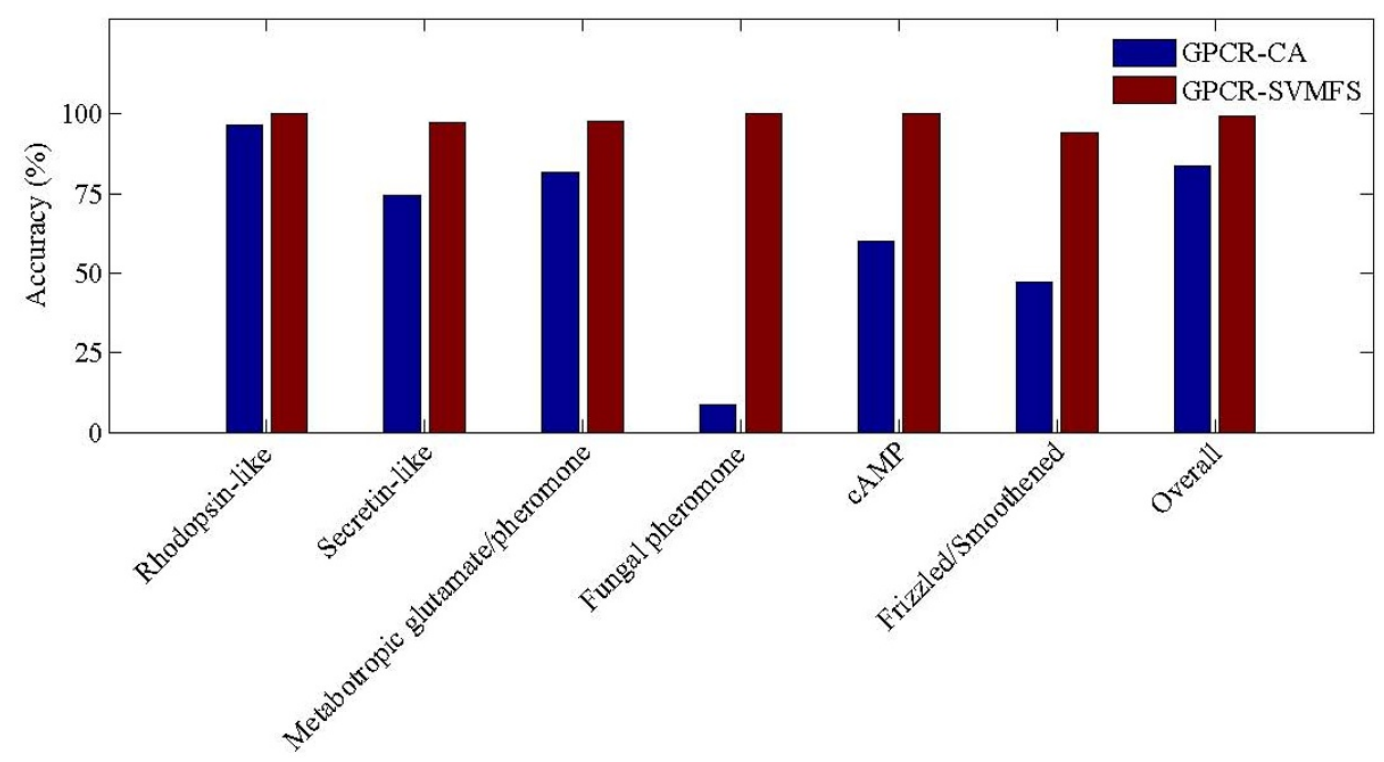

Figure 7 Comparison of different method by the jackknife test at family level. 
Table 3: The performance of GPCR-SVMFS and GPCRPred at superfamily level

\begin{tabular}{ccccc}
\hline Method & Acc(\%) & Sen(\%) & Spe(\%) & MCC \\
\hline GPCRPred [14] & 99.50 & 98.60 & 99.80 & 0.9900 \\
GPCR-SVMFSa & 100.0 & 100.0 & 100.0 & 1.0000 \\
\hline
\end{tabular}

a In order to consistent with evaluation method of GPCRPred, 5 -fold cross-validation is utilized.

Table 4: The performance of GPCR-SVMFS and GPCRPred at family level

\begin{tabular}{|c|c|c|c|c|c|c|}
\hline \multirow[t]{2}{*}{ Method } & \multicolumn{6}{|c|}{$Q_{i} / Q(\%)$} \\
\hline & Class A & Class B & Class C & Class D & Class E & Overall \\
\hline GPCRPred [14] & 98.10 & 85.70 & 81.30 & 36.40 & 100.0 & 97.30 \\
\hline GPCR-SVMFSa & 100.0 & 100.0 & 100.0 & 81.82 & 100.0 & 99.74 \\
\hline
\end{tabular}

a In order to consistent with evaluation method of GPCRPred, 2-fold cross-validation is utilized.

Table 5: The performance of GPCR-SVMFS and GPCRPred at subfamily level

\begin{tabular}{cccc}
\hline Class A subfamilies & Number of proteins & Q $/$ Q (\%) \\
& & GPCRPred [14] & GPCR-SVMFS \\
\cline { 2 - 4 } & & 99.10 & 100.0 \\
Amine & 221 & 99.70 & 99.21 \\
Peptide & 381 & 100.0 & 100.0 \\
Hormone & 25 & 98.90 & 99.45 \\
Rhodopsin & 183 & 100.0 & 100.0 \\
Olfactory & 87 & 100.0 & 100.0 \\
Prostanoid & 38 & 85.40 & 93.75 \\
Nucleotide & 48 & 100.0 & 90.91 \\
Cannabis & 11 & 100.0 & 100.0 \\
Platelet activating factor & 4 & 100.0 & 100.0 \\
Gonadotrophin releasing hormone & 10 & 85.70 & 85.71 \\
Thyrotropin releasing hormone & 7 & 100.0 & 100.0 \\
Melatonin & 13 & 33.30 & 76.47 \\
Viral & 17 & 98.80 & 100.0 \\
Lysospingolipids & 1054 & 97.30 & 98.77 \\
Overall & 9 & & \\
\hline
\end{tabular}

\footnotetext{
a In order to consistent with evaluation method of GPCRPred, 2 -fold cross-validation is utilized.
} 
Table 6: The prediction power of GPCR-SVMFS to independent dataset at family level

\begin{tabular}{cccc}
\hline GPCR family & Number of proteins & Number of correct prediction & \\
\hline Rhodopsin-like & 20290 & 19510 & $\mathbf{Q}_{\boldsymbol{i}} / \mathbf{Q}(\%)$ \\
Metabotropic & 1194 & 1024 & 96.16 \\
Secretin-like & 1484 & 1017 & 85.76 \\
Overall & 22972 & 21551 & 68.53 \\
\hline
\end{tabular}

Table 7: The prediction power of GPCR-SVMFS to independent dataset at subfamily level

\begin{tabular}{|c|c|c|c|}
\hline GPCR subfamily & Number of proteins & Number of correct prediction & $Q_{i} / Q(\%)$ \\
\hline Amine & 1840 & 1476 & 80.22 \\
\hline Peptide & 4169 & 3090 & 74.12 \\
\hline Rhodopsin & 1376 & 1208 & 87.79 \\
\hline Olfactory & 9977 & 9075 & 90.96 \\
\hline Nucleotide & 576 & 315 & 54.69 \\
\hline Overall & 17938 & 15164 & 84.54 \\
\hline
\end{tabular}

\section{Additional material}

Additional file 1 Eight amino acid properties extracted from AAlndex database are selected to compute autocorrelation features.

Additional file 2 The values of seven properties are obtained from AAlndex database, and the definitions of amino acids attributed to each group are shown in the table.

Additional file $\mathbf{3}$ More explain and an example to compute probability.

Additional file 4 Deorphan dataset. The file contains orphan protein's Swiss-Prot accession number and its corresponding sequence.

Additional file $\mathbf{5}$ Rhodopsin-like dataset. The file contains rhodopsinlike protein's Swiss-Prot accession number and its corresponding sequence.

Additional file $\mathbf{6}$ Metabotropic dataset. The file contains metabotropic protein's Swiss-Prot accession number and its corresponding sequence.

Additional file $\mathbf{7}$ Secretion-like dataset. The file contains secretion-like protein's Swiss-Prot accession number and its corresponding sequence.

Additional file $\mathbf{8}$ Amine dataset. The file contains amine protein's SwissProt accession number and its corresponding sequence.

Additional file $\mathbf{9}$ Peptide dataset. The file contains peptide protein's Swiss-Prot accession number and its corresponding sequence.

Additional file 10 Rhodopsin dataset. The file contains rhodopsin protein's Swiss-Prot accession number and its corresponding sequence.

Additional file 11 Olfactory dataset. The file contains olfactory protein's Swiss-Prot accession number and its corresponding sequence.

Additional file 12 Nucleotide dataset. The file contains nucleotide protein's Swiss-Prot accession number and its corresponding sequence.

\section{Authors' contributions}

$\mathrm{ZCL}$ conceived the idea and developed the programs, carries out the analyses and drafted the manuscript. XZ contributed to the ideas on overall design, implementation. ZD carried out data acquisition, guided the implementation of the work. XYZ supervised the design of the system, and advised on the manuscript preparation. All authors read and approved the final manuscript.

\section{Acknowledgements}

The authors acknowledge financial support from the National Natural Science Foundation of China (No. 20975117; 20805059), Ph.D. Programs Foundation of Ministry of Education of China (No. 20070558010) and Natural Science Foundation of Guangdong Province (No. 7003714)

\section{Author Details}

School of Chemistry and Chemical Engineering, Sun Yat-Sen University, Guangzhou 510275, PR China

Received: 18 December 2009 Accepted: 16 June 2010

Published: 16 June 2010

References

1. Papasaikas PK, Bagos PG, Litou ZI, Hamodrakas SJ: A novel method for GPCR recognition and family classification from sequence alone using signatures derived from profile hidden markov models. SAR QSAR Environ Res 2003, 14:413-420.

2. Gao QB, Wang ZZ: Classification of G-protein coupled receptors at four levels. Protein Eng Des Sel 2006, 19:511-516.

3. Eo HS, Choi JP, Noh SJ, Hur CG, Kim W: A combined approach for the classification of $\mathrm{G}$ protein-coupled receptors and its application to detect GPCR splice variants. Comput Biol Chem 2007, 31:246-256.

4. Baldwin JM: Structure and function of receptors coupled to $G$ proteins. Curr Opin Cell Biol 1994, 6:180-190.

5. Lefkowitz RJ: The superfamily of heptahelical receptors. Nat Cell Biol 2000, 2:e133-e136.

6. Qian B, Soyer OS, Neubig RR, Goldstein RA: Depicting a protein's two faces: GPCR classification by phylogenetic tree-based HMMs. FEBS Lett 2003, 554:95-99.

7. Chou KC, Elord DW: Bioinformatical analysis of G-protein-coupled receptors. J Proteome Res 2002, 1:429-433.

8. Karchin R, Karplus K, Haussler D: Classifying G-protein coupled receptors with support vector machines. Bioinformatics 2002, 18:147-159.

9. Hebert TE, Bouvier M: Structural and functional aspects of $\mathrm{G}$ proteincoupled receptor oligomerization. Biochem Cell Biol 1998, 76:1-11.

10. Xiao X, Wang P, Chou KC: GPCR-CA: A cellular automaton image approach for predicting G-protein-coupled receptor functional classes. J Comput Chem 2009, 30:1414-1423. 
11. Lapinsh M, Prusis P, Uhlen S, Wikberg JES: Improved approach for proteochemometrics modeling: application to organic compoundamino $\mathrm{G}$ protein-coupled receptor interactions. Bioinformatics 2005, 21:4289-4296.

12. Lapinsh M, Gutcaits A, Prusis P, Post C, Lundstedt T, Wikberg JE: Classification of $\mathrm{G}$-protein coupled receptors by alignmentindependent extraction of principal chemical properties of primary amino acid sequences. Protein Sci 2002, 11:795-805

13. Inoue $Y$, Ikeda M, Shimizu T: Proteome-wide classification and identification of mammalian-type GPCRs by binary topology pattern. Comput Biol Chem 2004, 28:39-49.

14. Bhasin M, Raghava GPS: GPCRpred: an SVM-based method for prediction of families and subfamilies of G-protien coupled receptors. Nucleic Acids Res 2004, 32:W383-W389.

15. Gupta R, Mittal A, Singh K: A novel and efficient technique for identification and classification of gpcrs. IEEE Trans Inf Technol Biomed 2008, 12:541-548.

16. Bhasin M, Raghava GP: GPCRsclass: a web tool for the classification of amine type of G-protein-coupled receptors. Nucleic Acids Res 2005, 33:W143-W147

17. Guo YZ, Li M, Lu M, Wen Z, Wang K, Li G, Wu J: Classifying G proteincoupled receptors and nuclear receptors on the basis of protein power spectrum from fast fourier transform. Amino Acids 2006, 30:397-402.

18. Papasaikas PK, Bagos PG, Litou ZI, Promponas VJ, Hamodrakas SJ: PREDGPCR: GPCR recognition and family classification server. Nucleic Acids Res 2004, 32:W380-W382.

19. Elrod DW, Chou KC: A study on the correlation of G-protein-coupled receptor types with amino acid composition. Protein Eng 2002, 15:713-715.

20. Chou KC: Prediction of G-protein-coupled receptor classes. J Proteome Res 2005, 4:1413-1418.

21. Khan A, Khan MF, Choi TS: Proximity based GPCRs prediction in transform domain. Biochem Biophys Res Commun 2008, 371:411-415.

22. Huang Y, Cai J, Ji L, Li Y: Classifying G-protein coupled receptors with bagging classification tree. Comput Biol Chem 2004, 28:275-280.

23. Davies MN, Secker A, Freitas AA, Mendao M, Timmis J, Flower DR: On the hierarchical classification of $\mathrm{G}$ protein-coupled receptors. Bioinformatics 2007, 23:3113-3118.

24. Wen Z, Li M, Li Y, Guo Y, Wang K: Delaunay triangulation with partial least squares projection to latent structures: a model for $\mathrm{G}$-protein coupled receptors classification and fast structure recognition. Amino Acids 2007, 32:277-283.

25. Guo J, Chen H, Sun Z, Lin Y: A novel method for protein secondary structure prediction using dual-layer SVM and profiles. Proteins 2004, 54:738-743.

26. Kumar M, Bhasin M, Natt NK, Raghava GP: BhairPred: prediction of $\beta$ hairpins in a protein from multiple alignment information using ANN and SVM techniques. Nucleic Acids Res 2005, 33:W154-W159.

27. Chou KC, Cai YD: Using functional domain composition and support vector machines for prediction of protein subcellular location. J Biol Chem 2002, 277:45765-45769.

28. Hua S, Sun Z: Support vector machine approach for protein subcellular localization prediction. Bioinformatics 2001, 17:721-728

29. Cai YD, Zhou GP, Chou KC: Support vector machines for predicting membrane protein types by using functional domain composition. Biophys J 2003, 84:3257-3263.

30. Chou KC: Prediction of protein cellular attributes using pseudo-amino acid composition. Proteins 2001, 43:246-255.

31. Qiu JD, Huang JH, Liang RP, Lu XQ: Predction of G-protein-coupled receptor classes based on the concept of Chou's pseudo amino acid composition: an approach from discrete wavelet transform. Anal Biochem 2009, 390:68-73.

32. Lin WZ, Xiao X, Chou KC: GPCR-GIA: a web-server for identifying Gprotein coupled receptors and their families with grey incidence analysis. Protein Eng Des Sel 2009, 22:699-705.

33. Xiao X, Lin WZ, Chou KC: Using grey dynamic modeling and pseudo amino acid composition to predict protein structural classes. J Comput Chem 2008, 29:2018-2024

34. Xiao X, Wang P, Chou KC: Predicting protein structural classes with pseudo amino acid composition: an approach using geometric moments of cellular automaton image. J Theor Biol 2008, 254:691-696.
35. Xiao X, Wang P, Chou KC: Predicting protein quaternary structural attribute by hybridizing functional domain composition and pseudo amino acid composition. J App/ Crystallogr 2009, 42:169-173.

36. Xiao X, Lin WZ: Application of protein grey incidence degree measure to predict protein quaternary structural types. Amino Acids 2009, 37:741-749.

37. Chen $C$, Chen LX, Zou XY, Cai PX: Predicting protein structural class based on multi-features fusion. J Theor Biol 2008, 253:388-392

38. Gao QB, Ye XF, Jin ZC, He J: Improving discrimination of outer membrane proteins by fusing different forms of pseudo amino acid composition. Analy Biochem 2010, 398:52-59.

39. Gao QB, Jin ZC, Ye XF, Wu C, He J: Prediction of unclear receptors with optimal pseudo amino acid composition. Anal Biochem 2009, 387:54-59.

40. Ma S, Huang J: Penalized feature selection and classification in bioinformatics. Brief Bioinform 2008, 9:392-403.

41. Xiong $M$, Fang $X$, Zhao J: Biomarker identification by feature wrappers. Genome Res 2001, 11:1878-1887.

42. Jirapech-Umpai T, Aitken S: Feature selection and classification for microarray data analysis: evolutionary methods for identifying predictive genes. BMC Bioinformatics 2005, 6:148.

43. Ooi $\mathrm{CH}$, Tan P: Genetic algorithms applied to multi-class prediction for the analysis of gene expression data. Bioinformatics 2003, 19:37-44.

44. Li L, Weinberg CR, Darden TA, Pedersen LG: Gene selection for sample classification based on gene expression data: study of sensitivity to choice of parameters of the GA/KNN method. Bioinformatics 2001, 17:1131-1142.

45. Gevaert O, Smet FD, Timmerman D, Moreau Y, Moor BD: Predicting the prognosis of breast cancer by integrating clinical and microarray data with bayesian networks. Bioinformatics 2006, 22:e184-e190.

46. Liu H, Li J, Wong L: A comparative study on feature selection and classification methods using gene expression profiles and proteomic patterns. Genome inform 2002, 13:51-60.

47. Prados J, Kalousis A, Sanchez JC, Allard L, Carrette O, Hilario M: Mining mass spectra for diagnosis and biomarker discovery of cerebral accidents. Proteomics 2004, 4:2320-2332.

48. Li L, Umbach DM, Terry P, Taylor JA: Application of the GA/KNN method to SELDI proteomics data. Bioinformatics 2004, 20:1638-1640.

49. Ressom HW, Varghese RS, Drake SK, Hortin GL, Abdel-Hamid M, Loffredo C A: Goldman R: Peak selection from MALDI-TOF mass spectra using ant colony optimization. Bioinformatics 2007, 23:619-626.

50. Bhanot $\mathrm{G}$, Alexe G, Venkataraghavan B, Levine AJ: A robust metaclassification strategy for cancer detection from MS data. Proteomics 2006, 6:592-604

51. Peng $H$, Long $F$, Ding $C$ : Feature selection based on mutual information: criteria of max-dependency, max-relevance, and min-redundancy. IEEE Trans Pattern Anal Mach Intell 2005, 27:1226-1238.

52. JH: Adaptation in Natural and Artificial Systems. The University of Michigan Press, USA 1975

53. Horn F, Weare J, Beukers MW, Horsch S, Bairoch A, Chen W, Edvardsen O, Campagne F, Vriend G: GPCRDB: an information system for $\mathrm{G}$ proteincoupled receptors. Nucleic Acids Res 1998, 26:275-279.

54. Dubchak I, Muchnik I, Holbrook SR, Kim SH: Prediction of protein folding class using global description of amino acid sequence. Proc Nat/ Acad SciUSA 1995, 92:8700-8704.

55. Kawashima S, Kanehisa M: AAindex: amino acid index database. Nucleic Acids Res 2000, 28:374

56. Lim VI: Algorithms for prediction of a-helical and $\beta$-structural regions in globular proteins. J Mol Biol 1974, 88:873-894.

57. Schiffer M, Edmundson AB: Use of helical wheels to represent the structures of proteins and to identify segments with helical potential. Biophys J 1967, 7:121-136.

58. Rose GD, Geselowitz AR, lesser GJ, Lee RH, Zehfus MH: Hydrophobicity of amino acid residues in globular proteins. Science 1985, 229:834-838.

59. Zhang HH, Ahn J, Lin X, Park C: Gene selection using support vector machines with non-convex penalty. Bioinformatics 2006, 22:88-95.

60. Segal MR, Dahlquist KD, Conklin BR: Regression approaches for microarray data analysis. J Comput Biol 2003, 10:961-980.

61. Saeys $Y$, Inza I, Larranaga P: A review of feature selection techniques in bioinformatics. Bioinformatics 2007, 23:2507-2517. 
62. LV QZ, Shen GL, Yu RQ: A chaotic approach to maintain the pupulation diversity of genetic algorithm in network training. Comput Biol Chem 2003, 27:363-371.

63. Li ZC, Zhou XB, Lin YR, Zou XY: Prediction of protein structure class by coupling improved genetic algorithm and support vector machine. Amino Acids 2008, 35:580-590.

64. Chang CC, Lin CJ: LIBSVM: a library for support vector machines. [http:/ /www.csie.ntu.edu.tw/ cjlin/libsvm].

65. Matthews BW: Comparison of predicted and observed secondary structure of T4 phage lysozyme. Biochem Biophys Acta 1975, 405:442-451.

\section{doi: 10.1186/1471-2105-11-325}

Cite this article as: Li et al., Classification of G-protein coupled receptors based on support vector machine with maximum relevance minimum redundancy and genetic algorithm BMC Bioinformatics 2010, 11:325

Submit your next manuscript to BioMed Central and take full advantage of:

- Convenient online submission

- Thorough peer review

- No space constraints or color figure charges

- Immediate publication on acceptance

- Inclusion in PubMed, CAS, Scopus and Google Scholar

- Research which is freely available for redistribution

Submit your manuscript at www.biomedcentral.com/submit
() BioMed Central 